\title{
Intravenous administration of triptonide attenuates CFA-induced pain hypersensitivity through inhibiting AKT signaling pathway in mice
}

Yue-Juan Ling

Nantong University

Ting-Yu Ding

Nantong University

Yong-Jing Gao

Nantong University

Bao-Chun Jiang ( $\nabla$ jiangbaochun@ntu.edu.cn )

Nantong University https://orcid.org/0000-0002-9053-887X

Research article

Keywords: Triptonide, AKT, DRG, Complete Freund's adjuvant, Inflammatory pain

Posted Date: January 3rd, 2020

DOI: https://doi.org/10.21203/rs.2.19974/v1

License: (c) (i) This work is licensed under a Creative Commons Attribution 4.0 International License.

Read Full License 


\section{Abstract}

Background: Triptonide (TPN) is a major component of Tripterygium wilfordii Hook.f., and reportedly has anti-inflammatory and neuroprotective effects. Recent studies have demonstrated that the phosphatidylinositol 3-kinase (PI3K)/AKT pathway plays an important role in the pathogenesis of inflammatory pain. Here we investigated the anti-nociceptive effect of systemic treatment with TPN on mouse models of chronic inflammatory pain and explored possible mechanisms.

Results: Unilateral hind paw injection of complete Freund's adjuvant (CFA) induced paw edema and persistent pain hypersensitivity. Intravenous treatment with TPN attenuated CFA-induced paw edema, mechanical allodynia, and thermal hyperalgesia. Western blotting and immunofluorescence results showed that CFA induced AKT activation in the dorsal root ganglion (DRG) neurons, which was inhibited by TPN treatment. Furthermore, TPN treatment inhibited mRNA increase of proinflammatory cytokines [tumor necrosis factor-a (TNF-a), interleukin 1 beta (IL-1 $\beta$ ), and Interleukin 6 (IL-6)] induced by CFA.

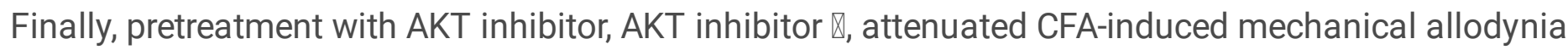
and thermal hyperalgesia, and decreased the mRNA expression of pro-inflammatory cytokines.

Conclusions: These data indicate that TPN attenuates CFA-induced pain potentially via inhibiting AKTmediated pro-inflammatory cytokines production in DRG. TPN may be used for the treatment of chronic inflammatory pain.

\section{Background}

Triptolide (TPL) is the main bioactive component of the herbal medicine Tripterygium wilfordii Hook. $f$. and has been used as a part of systematic medication to treat a wide variety of diseases, including cancer, lupus, rheumatoid arthritis, Alzheimer's disease, Parkinson's disease, and rheumatoid arthritis [13]. TPL can effectively relieve neuropathic pain by inhibiting the activation of microglia and astrocytes in the spinal dorsal horn $[4,5]$. TPL also attenuates cancer pain via suppressing the up-regulation of Chemokine (C-C motif) ligand 5 and histone deacetylases in the spinal glial cells [6, 7]. In addition, TPL inhibits the activation of extracellular signal-regulated kinase (ERK) pathway and the production of inflammatory cytokines in the spinal cord dorsal horn induced by inflammation [8]. Recently, TPL was reported to have a potent anti-depressive function by its influences on hippocampal neuroinflammation in a rat model of depression comorbidity of chronic pain [2]. However, clinical reports showed that TPL exposure resulted in the injury of some organs, including liver, kidney, heart, testes, and ovary in humans [9-11]. Severe hepatotoxicity was also shown after TPL exposure in animals $[9,12,13]$.

Triptonide (TPN) is another bioactive component of Tripterygium wilfordii Hook. f.. It was reported that TPN does not induce liver toxicity in animals $[1,3,14]$. The differences between the chemical structures of TPL and TPN are the substituent groups at C-14 position in which TPL is with C-14-hydroxyl and TPN is with C-14-carbonyl. The metabolomics study shows that the hydroxyl group at C-14 in the molecular 
structure of TPL plays an important role in its hepatotoxicity $[14,15]$. TPN acts as a novel potent anticancer drugs with low toxicity [16-18].

Phosphoinositide 3-kinase/protein kinase B/the mammalian target of rapamycin (PI3K/AKT/mTOR) pathway could be activated in DRG neurons and spinal glial cells in different pain models [19, 20]. Upregulating spinal mTOR activity by knocking down the mTOR-negative regulator reduced morphine analgesia and produced pain hypersensitivity [21]. Recent studies showed that TPN can inactivate AKT and induce caspase-dependent death in cervical cancer cells [22]. TPN has also been shown to remarkably diminish both ERK and AKT signaling pathways in lymphoma [1]. In addition, TPN can efficaciously suppress PCa growth in vitro and in vivo via inhibiting the phosphorylation of mTOR and the activities of related downstream signaling pathways [3]. Although the analgesic function of TPL has been studied, whether TPN has an analgesic effect and the underlying mechanisms on inflammatory pain remains unknown.

In this study, we investigated whether systemic treatment with TPN can attenuate nocifensive behaviors in complete Freund's adjuvant (CFA)-induced inflammatory pain model. We also explored the possible analgesic mechanisms of TPN by assaying the activation of AKT pathway and the production of inflammatory cytokines.

\section{Results}

\section{Triptonide attenuates CFA-induced inflammatory pain}

To test the anti-nociceptive effect of TPN in CFA-induced inflammatory pain, different doses of TPN or vehicle were intravenously injected daily for 5 consecutive days, with the first injection of TPN given at $1 \mathrm{~h}$ before CFA (Fig. 1A). On Day 1, the left hind paw volume of the CFA group was increased by 2 times compared to the baseline (Fig. 1B). Figure 1B showed that TPN at $0.5 \mathrm{mg} / \mathrm{kg}$ or $2.0 \mathrm{mg} / \mathrm{kg}$ resulted in a statistically significant decrease of paw swelling from 3 days to 5 days after CFA injection compared with untreated CFA mice $\left[\left(F_{2,76}=12.48\right.\right.$ and $\left.P=0.0003\right)$, Time $\left(F_{4,76}=9.34\right.$ and $\left.P<0.0001\right)$, and Time $x$ Treatment interaction $\left(F_{8,76}=1.56\right.$ and $\left.P=0.1525\right)$ ]. This revealed that TPN alleviated CFA-induced paw edema and has a strong anti-inflammatory effect.

For thermal hyperalgesia, an analysis of behavior data after TPN treatments by two-way ANOVA revealed a significant effect of Treatment $\left[\left(F_{2,76}=13.88\right.\right.$ and $\left.P=0.0002\right)$, Time $\left(F_{4,76}=40.92\right.$ and $\left.P<0.0001\right)$, and Time $\times$ Treatment interaction $\left(F_{8,76}=2.12\right.$ and $\left.\left.P=0.0442\right)\right]$. The Bonferroni post hoc tests showed that TPN at $2 \mathrm{mg} / \mathrm{kg}$ had no effect on CFA-induced thermal hyperalgesia in the first 2 days after CFA injection, but started to show a reversal effect at 3 days and maintained till 5 days (Fig. 1C). TPN at $0.5 \mathrm{mg} / \mathrm{kg}$ also significantly attenuated CFA-induced thermal hyperalgesia from 3 days to 4 days after CFA injection

(Fig. 1C). Meanwhile, TPN attenuated CFA-induced mechanical allodynia (Treatment, $F_{2,76}=7.94$ and $P<$ 0.0031 ; Time, $F_{4,76}=9.60$ and $P<0.0001$; Interaction, $F_{8,76}=2.92$ and $\left.P=0.0067\right)$. TPN at $2 \mathrm{mg} / \mathrm{kg}$ or at $0.5 \mathrm{mg} / \mathrm{kg}$ attenuated mechanical allodynia at 4 days and 5 days after CFA injection (Fig. 1D). These 
data suggest that repeated TPN administration attenuates CFA-induced pain hypersensitivity, especially thermal hyperalgesia

\section{TPN inhibits CFA-induced AKT activation in DRG}

To determine whether the analgesic effects of TPN were associated with inhibition of the AKT signaling pathways, we evaluated the expression level of phosphorylation of AKT (pAKT) in the DRG. Western blotting showed that PAKT was significantly increased at 5 days after CFA injection $(P<0.01)$. Pretreatment with TPN (2 mg/kg) significantly decreased CFA-induced pAKT upregulation $(P<0.01$, Fig. 2A and B). CFA injection with vehicle treatment increased pAKT-immunofluorescence (IF) 5 days after CFA injection ( $P<0.001$, Fig. $2 \mathrm{C}$ and 2D). In TPN-treated animals, both the number of pAKT positive cells and the intensity of pAKT-IF were significantly reduced $(P<0.001$, Fig. 2C and 2D), which was consistent with the Western blotting results. Double immunostaining further showed that PAKT was expressed in the DRG neurons (Fig. 2E).

TPN inhibits CFA-induced the increase of TNF- $a, I L-1 \beta$, and IL- 6 in the DRG

TNF-a, IL-1 $\beta$ and IL-6 are important proinflammatory cytokines in mediating peripheral sensitization and neuropathic pain [23-25]. To check whether the anti-nociceptive effect of TPN is associated with the downregulation of proinflammatory cytokines, we checked TNF-a, IL-1 $\beta$, and IL-6 expression in the DRG after repeated TPN administration. qPCR results showed that, compared with control animals, TNF-a, IL$1 \beta$, and IL- 6 mRNAs were significantly increased in animals after 5 days of CFA injection $(P<0.05, P<$ 0.01 , $P<0.05$, respectively, one-way ANOVA, Fig. 3. A-C). Both the dose of $0.5 \mathrm{mg} / \mathrm{kg}$ and $2.0 \mathrm{mg} / \mathrm{kg}$ TPN treatments significantly reduced the CFA-induced mRNA increases of TNF-a, IL-1 $\beta$, and IL-6 (0.05 mg/kg: $\mathrm{P}<0.05, \mathrm{P}<0.01, \mathrm{P}<0.05 ; 2.0 \mathrm{mg} / \mathrm{kg}$ : $\mathrm{P}<0.05, \mathrm{P}<0.001, \mathrm{P}<0.05$; respectively, one-way ANOVA, Fig. 3. AC) These data indicate the inhibitory effect of TPN on CFA-induced TNF-a, IL-1 $\beta$, and IL- 6 expression in the DRG.

TPN attenuates LPS-induced pAKT activation, and TNF- $a$, IL-1 $\beta$, and IL-6 expression in the ND7/23 Cells

To further confirm that TPN is able to attenuate PAKT activation in DRG neurons under inflammatory conditions, we used the classical exogenous toll-like receptor 4 ligand LPS to stimulate ND7/23 cells, a DRG neuron cell line, to mimic inflammatory conditions in vitro. We first checked pAKT expression after incubation with LPS $(1 \mu \mathrm{g} / \mathrm{mL})$ for 6 hours. Western blotting showed that LPS induced a rapid and dramatic increase of pAKT expression ( $P<0.001$, one-way ANOVA). Preincubation with TPN, 1 hour before LPS application, decreased pAKT expression by $43.2 \%(P<0.05$, one-way ANOVA, Fig. 4. A and B), which was consistent with the in vivo results (Fig. 2. A and B).

To examine whether TPN inhibit TNF-a, IL-1 $\beta$, and IL-6 expression via pAKT, we pretreated ND7/23 Cells with AKT inhibitor, AKT inhibitor $\nabla, 1 \mathrm{~h}$ before LPS application. As shown in Fig. 4C-E, the qRT-PCR results showed that AKT inhibitor $\nabla(1 \mu \mathrm{g} / \mathrm{mL})$ treatment blocked CFA-induced TNF-a, IL-1 $1 \beta$, and IL-6 increase $(P<$ $0.001, P<0.01$, and $P<0.001$ respectively, in one-way ANOVA). In consistent with AKT inhibitor $\nabla^{\prime} s$ effect, pretreatment with TPN $(1 \mu \mathrm{g} / \mathrm{mL})$ also significantly decreased LPS-induced TNF- $a$, IL-1 $\beta$, and IL-6 
upregulation $(P<0.01, P<0.05$, and $P<0.001$ respectively, one-way ANOVA). These data suggest that TPN is involved in pAKT-mediated upregulation of TNF-a, IL-1 $\beta$, and IL- 6 in activated ND7/23 cells by LPS.

AKT inhibitor attenuates CFA-induced pain hypersensitivity and upregulation of TNF- $\alpha$, IL-1 $\beta$, and IL- 6 in DRG

To define whether AKT is associated with CFA-induced pain hypersensitivity and the upregulation of inflammatory cytokines, we intrathecally injected the AKT inhibitor $(1 \mu \mathrm{g} / 10 \mu \mathrm{l}) 3$ days after CFA injection.

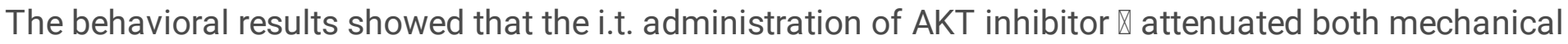
allodynia (Treatment, $F_{1,33}=5.35$ and $P=0.0411$; Time, $F_{3,33}=4.09$ and $P=0.0142$; Interaction, $F_{3,33}=$

3.26 and $P=0.0335$ ) and thermal hyperalgesia (Treatment, $F_{1,33}=5.18$ and $P=0.0113$; Time, $F_{3,33}=4.32$ and $\mathrm{P}=0.0113$; Interaction, $\mathrm{F}_{3,33}=6$ and $\mathrm{P}=0.0022$ ) at 3 and $6 \mathrm{~h}$, but not $24 \mathrm{~h}$ after injection (Fig. 5 . A and $B$ ). As shown in Fig. 5C-5E, AKT inhibitor $\otimes$ treatment for $3 \mathrm{~h}$ also significantly blocked the mRNA expression of TNF- $a$, and IL-1 $\beta(P<0.05)$. Taken together, these data suggest that AKT signaling pathway is involved in the development of CFA-induced inflammatory pain, and inhibition of AKT phosphorylation reduces the upregulation of inflammatory cytokines in the DRG.

\section{Discussion}

In this study, we investigated the anti-nociceptive effect of systemic injection of TPN on CFA-induced pain hypersensitivity, and explored possible mechanisms. Our results demonstrated that TPN administration attenuated CFA-induced edema and pain hypersensitivity. Meanwhile, TPN treatment inhibited the AKT signaling pathway and the expression of TNF- $\alpha$, IL-1 $\beta$, and IL- 6 in the DRG after CFA injection. AKT pathway acts as an upstream signaling pathway in CFA-induced upregulation of TNF-a, IL-1 $\beta$, and IL- 6 .

Previous studies showed that TPL was capable of preventing and attenuating neuropathic pain, cancer pain, and inflammatory pain in mice or rats via inhibiting central immune response [26], inhibiting the upregulation of histone deacetylases in spinal glial cells [7], or inhibiting spinal glia activation [8], but the analgesic effect of TPN in animal pain models is largely unknown. This is the first demonstration that single intravenous injection of TPN before CFA injection, persistently attenuated CFA-induced thermal hyperalgesia and mechanical allodynia. Pre-treatment with TPN dose-dependently inhibited CFA-induced thermal hyperalgesia. However, there is no significant difference in mechanical allodynia between the low-dose and high-dose TPN groups. This may result from the target molecules or signaling pathways of TPN play more important roles in thermal pain sensitivity processing. Besides, the injection of TPN could also effectively reduce paw edema caused by CFA. These data indicate the potential use of TPN in the treatment of inflammatory pain.

Although the PAKT pathway typically regulates cell growth and survival, increasing evidence indicates the involvement of this pathway in the development and maintenance of chronic pain $[27,28]$. Our data further showed that PAKT was predominantly expressed in DRG neurons after CFA injection. In agreement 
with our results, pAKT expression in neurons was found in the DRG following paclitaxel treatment [29]. Here, intraplantar injection of CFA induced pain hypersensitivity, and increased pAKT expression in the DRG, which was inhibited by TPN administration, suggesting that the anti-nociceptive effect of TPN may be mediated by PAKT-mediated peripheral sensitization in the DRG. In accord with our results, emerging evidence indicates that the expression of PAKT was suppressed by TPN on in vitro or in vivo models of different kinds of cancers $[1,3,22]$. How TPN inhibited PAKT signaling pathways remains to be defined.

TNF-a, IL-1 $\beta$ and IL- 6 are well-known pro-inflammatory cytokines that have been implicated in inflammatory pain [30]. Our data showed that the expression of TNF-a, IL-1 $\beta$, and IL- 6 was increased in DRG neurons after CFA injection, and was inhibited by TPN treatment. TNF-a was expressed in the majority of voltage-gated sodium channel (Nav) 1.3-positive or Nav1.8-positive neurons and up-regulated the expression of Nav1.3 and Nav1.8 in DRG neurons following peripheral nerve injury [23]. Cleaved IL-1 $\beta$ expression was significantly increased in small-sized DRG neurons after CFA injection into the hind paw [31]. IL-6 was up-regulated in the ipsilateral L4 and L5 DRG neurons and in the bilateral lumbar spinal cord following L5-ventral root transaction and contributed to the development of neuropathic pain [25]. Therefore, TPN may attenuate pain hypersensitivity via the inhibition of TNF- $\alpha$, IL-1 $\beta$, and IL-6-mediated neuroinflammation.

Pharmacological inhibition of pAKT inhibited TNF-a, IL-1 $\beta$, and IL- 6 production. Previous studies have demonstrated that inhibition of pAKT pathway prevented the LPS-induced expression of TNF-a in human bronchial epithelial cells [32]. Diesel exhaust particles exposure can activate the AKT signaling pathway, and further up-regulate IL-1 $\beta$ protein expression in primary human bronchial epithelial cells [32]. Studies from Caco-2 cells and fibroblast-like synoviocytes showed that IL-17-mediated induction of IL-6 was transduced via activation of AKT and NF-KB [33], while mitogen-activated protein kinase (MAPK) are not likely to participate in the process [29]. It is highly likely that the p-AKT pathway acts as an upstream signaling pathway up-regulated the expression of cytokines TNF-a, IL-1 $\beta$, and IL- 6 after CFA.

\section{Conclusion}

Our present study demonstrated that intravenous treatment with TPN significantly attenuated CFAinduced pain hypersensitivity, which was associated with decreased TNF-a, IL-1 $\beta$, and IL- 6 expression, and decreased pAKT activation in DRG neurons. These data suggest that TPN could attenuate inflammatory pain via the inhibition of pAKT/TNF-a-IL-6-IL-1 $\beta$ signaling pathway axis in DRG neurons. TPN may be of therapeutic value in inflammatory pain.

\section{Material And Methods}

\section{Animals}

Adult ICR male mice (6 to 8 weeks) were obtained from the Animal Care and Use Committee of Nantong University. Animals were kept under a $12 / 12 \mathrm{~h}$ light/dark cycle at a temperature of $23 \pm 2{ }^{\circ} \mathrm{C}$, humidity 
$(50 \%-60 \%)$ with free-feeding. All behavioral experiments were performed between 9 am and $6 \mathrm{pm}$. All in vivo studies were performed in accordance with the UK Animals Scientifc Procedures Act (1986) and were approved by the Animal Care and Use Committee of Nantong University.

\section{CFA pain model and drug administration}

The animals were randomly assigned to three groups of mice. An experimental timeline is shown in Fig. 1A. Inflammatory pain was induced by intraplantar injection of $20 \mu \mathrm{L}$ CFA (100\%; Sigma-Aldrich) in the left hind paws under brief anesthesia with isofluorane (2\%; RWD Life Science, Shenzhen, China). Each group, respectively, received a single dose intravenous injections once a day for 5 days through the tail vein as follows: Group CFA $(n=7)$ : Saline $0.9 \%$ at a TPN equivalent volume; Group CFA + TPN $(0.5$ $\mathrm{mg} / \mathrm{kg})(\mathrm{n}=7)$ : TPN at a dose of $0.5 \mathrm{mg} / \mathrm{kg}$ and Group CFA + TPN $(2.0 \mathrm{mg} / \mathrm{kg})(\mathrm{n}=8):$ TPN at a dose of $2 \mathrm{mg} / \mathrm{kg}$. The first intravenous injections were performed 1 hour before CFA treatment.

\section{Paw edema measurement}

Paw volumes were measured by plethysmometry (Ugo Basile Plethysmometer, Comerio, Italy) before CFA injections, and 1, 2, 3, 4, and 5 days after TPN administration. Edema was expressed by paw volume (ml).

\section{Behavioral analysis}

For the von Frey test, the animals were put in boxes on an elevated metal mesh floor daily for at least $2 \mathrm{~d}$ before baseline testing and allowed $30 \mathrm{~min}$ for habituation before the examination. The plantar surface of the left hind paw was stimulated with a series of von Frey hairs with logarithmically incrementing stiffness (0.02-2.56 g, Stoelting). The $50 \%$ paw withdrawal threshold was determined using Dixon's updown method. For the Hargreaves test, the animals were put in a plastic box placed on a glass plate, and the plantar surface was exposed to a beam of radiant heat through a transparent glass surface (Life Science). The baseline latencies were adjusted to 10-14 $\mathrm{s}$ with a maximum of $20 \mathrm{~s}$ as a cutoff to prevent potential injury. All the behavioral experimenters were done by individuals who were blinded to the treatment of the mice.

\section{Cell cultures}

Mouse neuroblastoma/rat DRG neuron hybrid ND7/23 (RRID:CVCL_4259) cells were grown under standard cell culture conditions $\left(5 \% \mathrm{CO}_{2}, 37^{\circ} \mathrm{C}\right)$ in High Glucose Dulbecco's Modified Eagle Medium (DMEM, Gibco) supplemented with $10 \%$ fetal bovine serum and $100 \mathrm{U} / \mathrm{ml}$ Penicillin-Streptomycin. 
ND7/23 cells were incubated with $1 \mu \mathrm{g} / \mathrm{mL}$ Lipopolysaccharides (LPS, Sigma-Aldrich, St. Louis, MO, USA) for $6 \mathrm{~h}$. The treatment of the $1 \mu \mathrm{g} / \mathrm{mL}$ TPN (Chengdu Mansite Biotechnology, Sichuan, China) or the treatment of $1 \mu \mathrm{g} / \mathrm{mL}$ AKT inhibitor IV (Sigma-Aldrich) was started $1 \mathrm{~h}$ prior to LPS treatment.

\section{Western blotting}

Western blotting analysis of PAKT levels were performed using samples collected from four experimental groups (Naive $\mathrm{n}=3$, CFA $\mathrm{n}=3$, CFA + TPN $(0.5 \mathrm{mg} / \mathrm{kg}), \mathrm{n}=3$, CFA + TPN $(2 \mathrm{mg} / \mathrm{kg}), \mathrm{n}=3)$. The tissues or cells were homogenized in a lysis buffer containing protease and phosphatase inhibitors (Sigma-Aldrich) and $30 \mathrm{\mu g}$ of proteins were loaded for each lane and separated on SDS-PAGE gel (10\%). After the transfer, the blots were incubated overnight at $4{ }^{\circ} \mathrm{C}$ with the pAKT primary antibody (RRID:AB_2716452; rabbit, 1:1000; Cell Signaling Technology, USA). For loading control, the blots were probed with AKT primary antibody (RRID:AB_2225340; rabbit, 1:1000; Cell Signaling Technology, USA)

\section{Immunohistochemistry}

Animals were deeply anesthetized with isoflurane and perfused through the ascending aorta with saline followed by $4 \%$ paraformaldehyde with $0.1 \mathrm{M}$ phosphate buffer. After the perfusion, the L4-L5 DRG were removed and postfixed in the same fixative overnight. DRG $(8-15 \mu \mathrm{m})$ were cut in a cryostat and processed for immunofluorescence. DRG was first blocked with $1 \%$ bovine serum albumin for $2 \mathrm{~h}$ at room temperature, then incubated overnight at $4{ }^{\circ} \mathrm{C}$ with the pAKT primary antibody (RRID:AB_2716452; rabbit, 1:200; Cell Signaling Technology, USA), The sections were then incubated for $2 \mathrm{~h}$ at room temperature with Cy3-conjugated secondary antibodies (1:1,000; Jackson ImmunoResearch). The stained sections were examined with Leica SP8 Gated STED confocal microscope (Leica Microsystems, Wetzlar, Germany). The pAKT fluorescence intensity were measured by ImageJ.

\section{Real-time qPCR}

Total RNA was extracted from L4-6 DRG with the Trizol reagent (Invitrogen, Carlsbad, CA, USA). One microgram of total RNA was converted into CDNA using PrimeScript RT reagent kit (Takara, Shiga, Japan). qPCR analysis was performed with the Real-Time Detection System by SYBR green I dye detection (Takara). The cDNA was amplified using the following primers: GAPDH forward, 5 c-AAA TGG TGA AGG TCG GTG TGA AC-3c; GAPDH reverse, 5ל-CAA CAA TCT CCA CTT TGC CAC TG-3c; TNF-a forward, 5c-GTT CTA TGG CCC AGA CCC TCA C-3 $\dot{c}$; reverse, 5לे-GGC ACC ACT AGT TGG TTG TCT TTG$3 c$; IL-1 $\beta$ forward, 5c-TCC AGG ATG AGG ACA TGA GCA C-3c; reverse, 5ל-GAA CGT CAC ACA CCA GCA GGT TA-3द; IL-6 forward, 5ל-TAG TGG ATG CTA CCA AAC TGG A-3द;; reverse, 5ל-TGT GAC TCC AGC TTA TCT CTT G G-3ç. 


\section{Quantification and statistics}

All data were analyzed by researchers blinded to the reagents used. All data were analyzed by GraphPad Prism (version 5.01) and presented as mean \pm SEM. $P<0.05$ was considered statistically significant. Behavioural data were analysed using two-way repeated measures ANOVA. Western blotting, immunofluorescence density and qPCR data were compared using one-way ANOVA. Student's $t$-test (2tailed) were used to analyse qPCR data if only 2 groups were applied.

\section{Declarations}

\section{Abbreviations}

TPN: Triptonide; PI3K: Phosphoinositide 3-kinase; AKT: Protein kinase B; mTOR: The mammalian target of rapamycin; CFA: complete Freund's adjuvant; DRG: dorsal root ganglion; TNF-a: Tumor Necrosis Factor Alpha; IL-1 $\beta$ : Interleukin 1 beta; IL-6: Interleukin 6; TPL: Triptolide; ERK: extracellular signal-regulated kinase; LPS: Lipopolysaccharides; GAPDH: glyceraldehyde 3-phosphate dehydrogenase; Nav: voltagegated sodium channel;

\section{Acknowledgments}

We would like to thank Fu-Lu Dong for the support and advice in the research.

\section{Author contributions}

YJL performed immunostaining, Western blotting, and behavioral experiments. TYD performed part of the behavioral experiments. BCJ and LYJ analyzed the data. BCJ conceived the project and coordinated and supervised the experiments. BCJ, YJG and YJL wrote the paper.

\section{Funding}

This work was funded by the grants from the National Natural Science Foundation of China (NSFC 81771197, 81971054, 81300954 and 81400915), the Natural Science Foundation of Jiangsu Province (BK20171255), the Six talent peaks project in Jiangsu Province (SWYY-070). 
Availability of data and materials

The datasets used and/or analyzed during the current study available from the corresponding author on reasonable request.

Ethics approval and consent to participate

All in vivo studies were performed in accordance with the UK Animals Scientifc Procedures Act (1986) and were approved by the Animal Care and Use Committee of Nantong University.

Consent for publication

Not applicable.

Competing of interests

The authors declare that they have no competing interests.

\section{References}

1. Yang P, Dong FL, Zhou QS: Triptonide acts as a novel potent anti-lymphoma agent with low toxicity mainly through inhibition of proto-oncogene Lyn transcription and suppression of Lyn signal pathway. Toxicol Lett 2017, 278:9-17.

2. Hu X, Dong Y, Jin X, Zhang C, Zhang T, Zhao J, Shi J, Li J: The novel and potent anti-depressive action of triptolide and its influences on hippocampal neuroinflammation in a rat model of depression comorbidity of chronic pain. Brain, behavior, and immunity 2017, 64:180-194.

3. Dong F, Yang P, Wang R, Sun W, Zhang Y, Wang A, Chen M, Chen L, Zhang C, Jiang M: Triptonide acts as a novel antiprostate cancer agent mainly through inhibition of mTOR signaling pathway. Prostate 2019, 79(11):1284-1293.

4. Tang J, Li Z-H, Ge S-N, Wang W, Mei X-P, Wang W, Zhang T, Xu L-X, Li J-L: The inhibition of spinal astrocytic JAK2-STAT3 pathway activation correlates with the analgesic effects of triptolide in the rat neuropathic pain model. Evidence-Based Complementary and Alternative Medicine 2012, 2012.

5. Huang Y, Zhu N, Chen T, Chen WJ, Kong JS, Zheng WB, Ruan JW: Triptolide Suppressed the Microglia Activation to Improve Spinal Cord Injury Through miR-96/IKK beta/NF-kappa B Pathway. Spine 2019, 44(12):E707-E714.

6. Hang LH, Li SN, Shao DH, Chen Z, Chen YF, Shu WW: Evidence for involvement of spinal RANTES in the antinociceptive effects of triptolide, a diterpene triepoxide, in a rat model of bone cancer pain. 
Basic \& clinical pharmacology \& toxicology 2014, 115(6):477-480.

7. Hu X-F, He X-T, Zhou K-X, Zhang C, Zhao W-J, Zhang T, Li J-L, Deng J-P, Dong Y-L: The analgesic effects of triptolide in the bone cancer pain rats via inhibiting the upregulation of HDACs in spinal glial cells. J Neuroinflamm 2017, 14(1):213.

8. Xu F, Li Y, Li S, Ma Y, Zhao N, Liu Y, Qian N, Zhao H, Li Y: Complete Freund's adjuvant-induced acute inflammatory pain could be attenuated by triptolide via inhibiting spinal glia activation in rats. journal of surgical research 2014, 188(1):174-182.

9. Mei Z, Wu Q, Hu S, Lib X, Yang X: Triptolide loaded solid lipid nanoparticle hydrogel for topical application. Drug development and industrial pharmacy 2005, 31(2):161-168.

10. Tang X, Wang C, Hsieh Y, Wang J, Han Z, Cong N, Ma R, Chi F: Triptolide induces toxicity in inner ear stem cells via promoting DNA damage. Toxicology in vitro : an international journal published in association with BIBRA 2019, 61:104597.

11. Xi C, Peng S, Wu Z, Zhou Q, Zhou J: Toxicity of triptolide and the molecular mechanisms involved. Biomed Pharmacother 2017, 90:531-541.

12. Li J, Shen F, Guan C, Wang W, Sun X, Fu X, Huang M, Jin J, Huang Z: Activation of Nrf2 protects against triptolide-induced hepatotoxicity. PloS one 2014, 9(7):e100685.

13. Vliegenthart ADB, Wei C, Buckley C, Berends C, de Potter CMJ, Schneemann S, Del Pozo J, Tucker C, Mullins JJ, Webb DJ et al: Characterization of Triptolide-Induced Hepatotoxicity by Imaging and Transcriptomics in a Novel Zebrafish Model. Toxicological sciences : an official journal of the Society of Toxicology 2017, 159(2):380-391.

14. Hu DD, Chen XL, Xiao XR, Wang YK, Liu F, Zhao Q, Li X, Yang XW, Li F: Comparative metabolism of tripolide and triptonide using metabolomics. Food Chem Toxicol 2018, 115:98-108.

15. Zhao Q, Zhang J-L, Li F: Application of Metabolomics in the Study of Natural Products. Natural products and bioprospecting 2018, 8(4):321-334.

16. Xiang S, Zhao Z, Zhang T, Zhang B, Meng M, Cao Z, Zhou Q: Triptonide effectively suppresses gastric tumor growth and metastasis through inhibition of the oncogenic Notch1 and NF-kappaB signaling pathways. Toxicology and applied pharmacology 2019:114870.

17. Wang SS, Lv Y, Xu XC, Zuo Y, Song Y, Wu GP, Lu PH, Zhang ZQ, Chen MB: Triptonide inhibits human nasopharyngeal carcinoma cell growth via disrupting Lnc-RNA THOR-IGF2BP1 signaling. Cancer letters 2019, 443:13-24.

18. Zhang B, Meng M, Xiang S, Cao Z, Xu X, Zhao Z, Zhang T, Chen B, Yang P, Li Y et al: Selective activation of tumor-suppressive MAPKP signaling pathway by triptonide effectively inhibits pancreatic cancer cell tumorigenicity and tumor growth. Biochem Pharmacol2019, 166:70-81.

19. Jiang S-P, Zhang Z-D, Kang L-M, Wang Q-H, Zhang L, Chen H-P: Celecoxib reverts oxaliplatin-induced neuropathic pain through inhibiting PI3K/Akt2 pathway in the mouse dorsal root ganglion. Experimental neurology 2016, 275:11-16.

20. Guo J-R, Wang H, Jin X-J, Jia D-L, Zhou X, Tao Q: Effect and mechanism of inhibition of $\mathrm{PI} 3 \mathrm{~K} / \mathrm{Akt} / \mathrm{mTOR}$ signal pathway on chronic neuropathic pain and spinal microglia in a rat model of 
chronic constriction injury. Oncotarget 2017, 8(32):52923.

21. Xu JT, Zhao JY, Zhao XL, Ligons D, Tiwari V, Atianjoh FE, Lee CY, Liang LL, Zang WD, Njoku D et al: Opioid receptor-triggered spinal mTORC1 activation contributes to morphine tolerance and hyperalgesia. J Clin Invest 2014, 124(2):592-603.

22. Kim MJ, Lee TH, Kim SH, Choi YJ, Heo J, Kim YH: Triptolide inactivates Akt and induces caspasedependent death in cervical cancer cells via the mitochondrial pathway. International journal of oncology 2010, 37(5):1177-1185.

23. He X-H, Zang Y, Chen X, Pang R-P, Xu J-T, Zhou X, Wei X-H, Li Y-Y, Xin W-J, Qin Z-H: TNF-a contributes to up-regulation of Nav1. 3 and Nav1. 8 in DRG neurons following motor fiber injury. PAIN® 2010, 151(2):266-279.

24. Copray J, Mantingh I, Brouwer N, Biber K, Küst B, Liem R, Huitinga I, Tilders F, Van Dam A-M, Boddeke $\mathrm{H}$ : Expression of interleukin-1 beta in rat dorsal root ganglia. Journal of neuroimmunology 2001, 118(2):203-211.

25. Wei X-H, Na X-D, Liao G-J, Chen Q-Y, Cui Y, Chen F-Y, Li Y-Y, Zang Y, Liu X-G: The up-regulation of IL-6 in DRG and spinal dorsal horn contributes to neuropathic pain following L5 ventral root transection. Experimental neurology 2013, 241:159-168.

26. Wang W, Mei XP, Chen L, Tang J, Li JL, Wu SX, Xu LX, Li YQ: Triptolide prevents and attenuates neuropathic pain via inhibiting central immune response. Pain Physician 2012, 15(6):E995-1006.

27. Zhuang Z-Y, Xu H, Clapham DE, Ji R-R: Phosphatidylinositol 3-kinase activates ERK in primary sensory neurons and mediates inflammatory heat hyperalgesia through TRPV1 sensitization. Journal of Neuroscience 2004, 24(38):8300-8309.

28. Chen S-P, Zhou Y-Q, Liu D-Q, Zhang W, Manyande A, Guan X-H, Tian Y-k, Ye D-W, Mohamed Omar D: PI3K/Akt pathway: a potential therapeutic target for chronic pain. Current pharmaceutical design 2017, 23(12):1860-1868.

29. Li D, Chen H, Luo X-H, Sun Y, Xia W, Xiong Y-C: CX3CR1-mediated Akt1 activation contributes to the paclitaxel-induced painful peripheral neuropathy in rats. Neurochemical research 2016, 41(6):13051314.

30. Cook AD, Christensen AD, Tewari D, McMahon SB, Hamilton JA: Immune cytokines and their receptors in inflammatory pain. Trends in immunology 2018, 39(3):240-255.

31. Matsuoka Y, Yamashita A, Matsuda M, Kawai K, Sawa T, Amaya F: The NLRP2 inflammasome in dorsal root ganglion as a novel molecular platform that produces inflammatory pain hypersensitivity. Pain 2019.

32. Wu W, Peden DB, McConnell R, Fruin S, Diaz-Sanchez D: Glutathione-S-transferase M1 regulation of diesel exhaust particle-induced pro-inflammatory mediator expression in normal human bronchial epithelial cells. Particle and fibre toxicology 2012, 9(1):31.

33. Hwang S-Y, Kim J-Y, Kim K-W, Park M-K, Moon Y, Kim W-U, Kim H-Y: IL-17 induces production of IL-6 and IL-8 in rheumatoid arthritis synovial fibroblasts via NF-KB-and PI3-kinase/Akt-dependent pathways. Arthritis Res Ther 2004, 6(2):R120. 


\section{Highlights}

TPN attenuates chronic inflammatory pain induced by CFA.

TPN inhibits CFA-induced AKT activation in the DRG neurons

TPN suppresses CFA-induced proinflammatory factors expression in the DRG

\section{Figures}

a

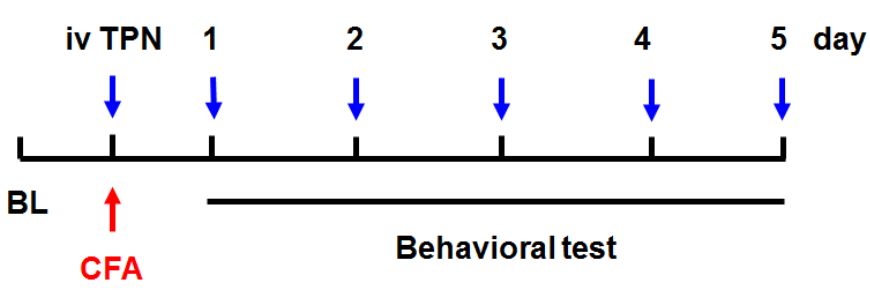

1h later

C

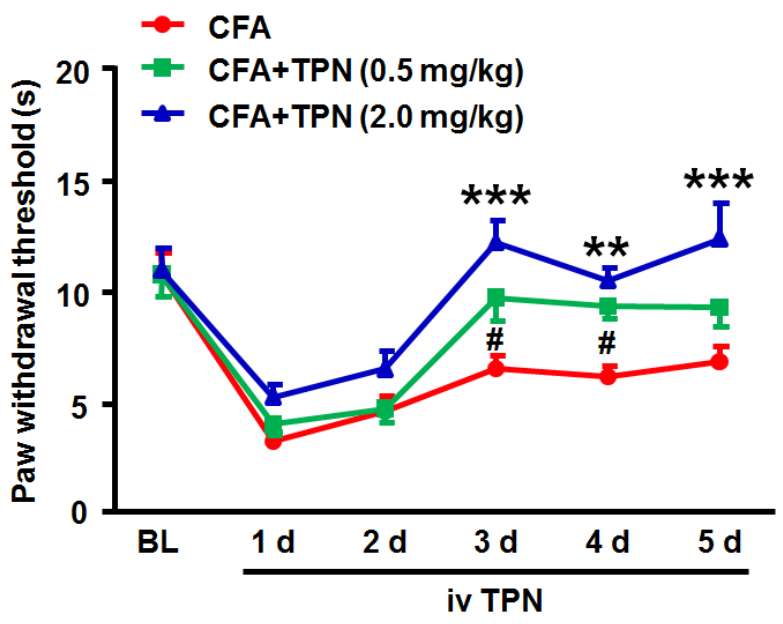

b

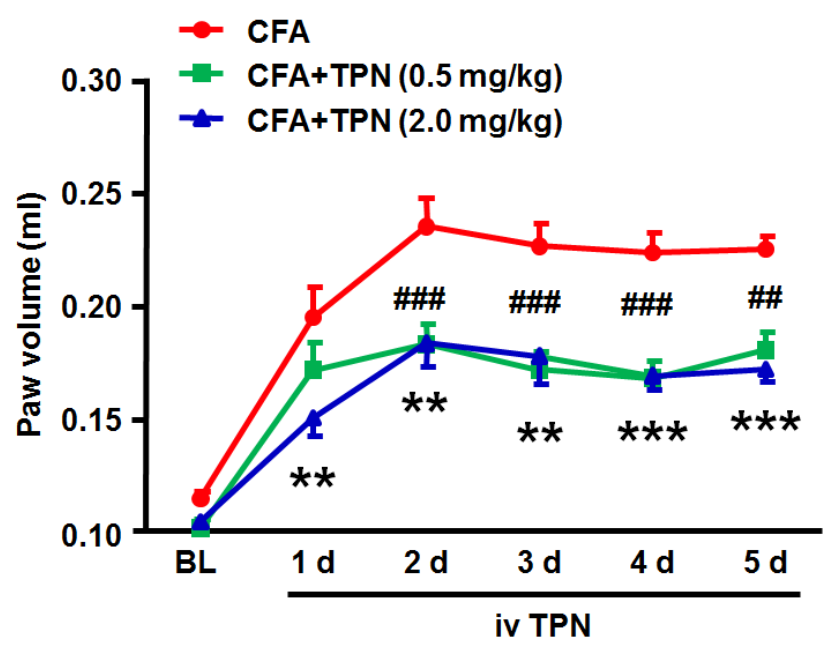

d

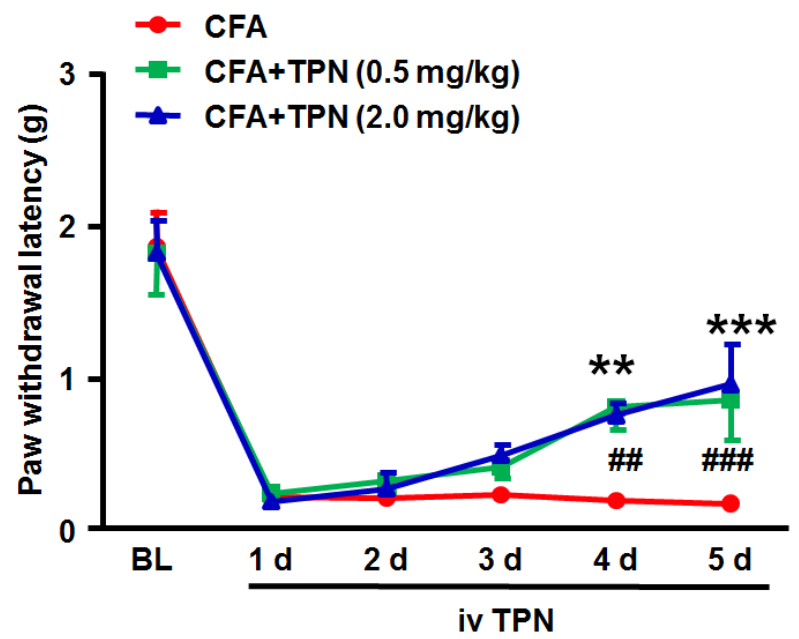

Figure 1

TPN treatment attenuates CFA-induced paw swelling, mechanical allodynia, and thermal hyperalgesia. (A) Schematic diagram of the timeline for CFA injection, drug treatment, and behavioral testing. (B) Paw volume measurements, (C) Thermal hyperalgesia, and (D) Mechanical allodynia of CFA-injected mice 
treated or untreated with TPN. \#P $<0.05$, \#\# < 0.01, \#\#\#P $<0.001$, low dose $(0.5 \mathrm{mg} / \mathrm{kg})$ compared to the vehicle; ${ }^{\star *} \mathrm{P}<0.01$, ${ }^{\star \star *} \mathrm{P}<0.001$, high dose $(2.0 \mathrm{mg} / \mathrm{kg})$ compared to the vehicle; 2 -way repeatedmeasures ANOVA followed by Bonferroni's test, $n=7 \sim 8$ mice/group.

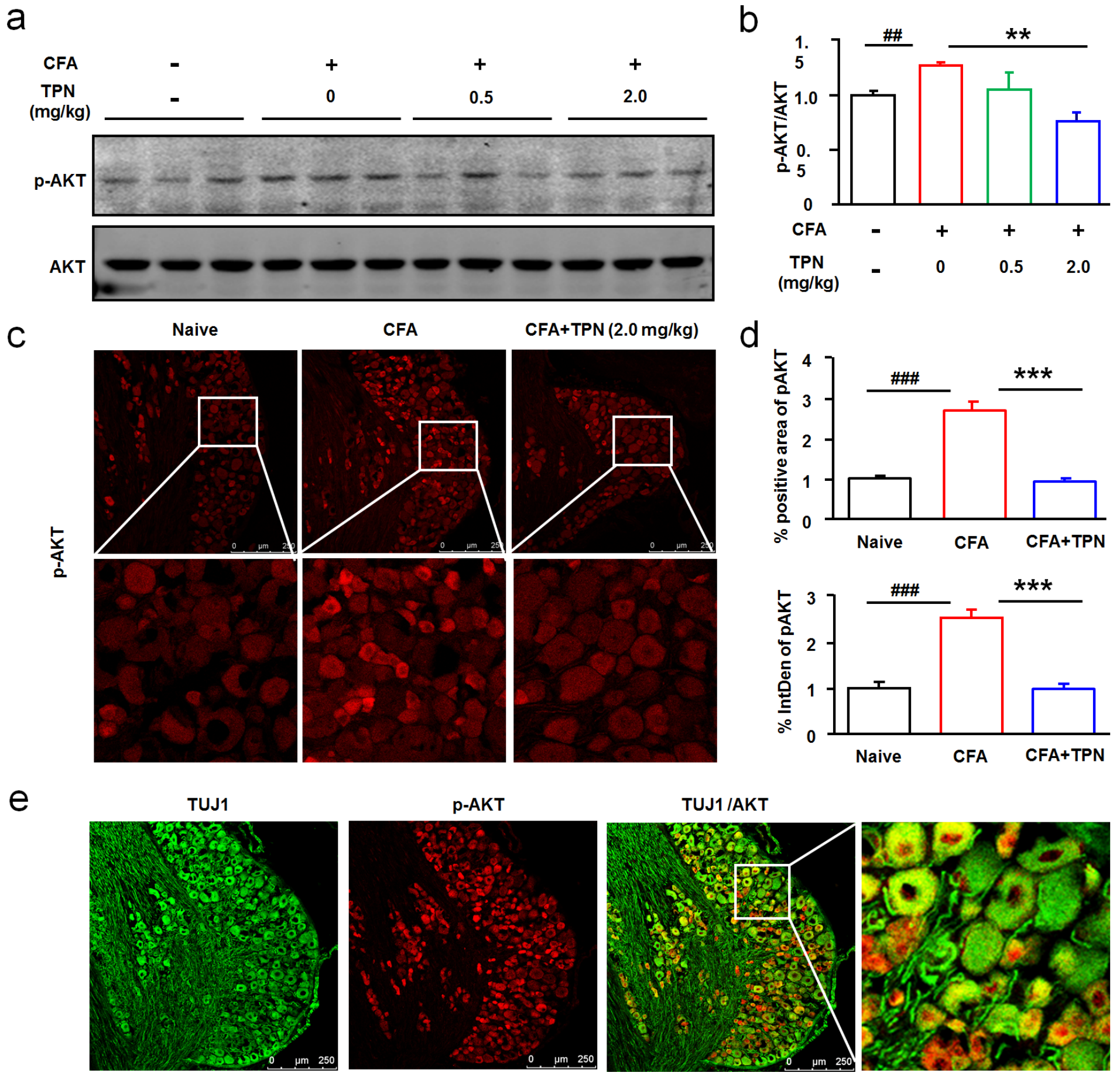

Figure 2

TPN treatment decreases pAKT expression in the DRG neurons. (A and B) pAKT expression of CFAinjected mice treated or untreated with TPN. PAKT expression was increased in DRG at 5 days after CFA injection, and recovered in CFA + TPN $(2 \mathrm{mg} / \mathrm{kg})$ animals. $\mathrm{n}=3$. \#\#P < 0.01, CFA group compared to Naïve; ${ }^{* *} \mathrm{P}<0.01$, high dose of TPN compared to CFA only group, one-way ANOVA; (C) Immunostaining of 
pAKT in the DRG; (D) Statistical data show the number of pAKT positive neurons and pAKT staining intensity in the DRG. $n=5$ mice/group. \#\#\#P < 0.001, CFA group compared to Naïve; ${ }^{* \star *} P<0.001, C F A+$ TPN compared to CFA group, one-way ANOVA. (E) Double staining of pAKT with a neuronal marker, $\beta$ tubulin (TUJ1) in the DRG at 5 days after CFA. Scale bars: $250 \mu \mathrm{m}$.

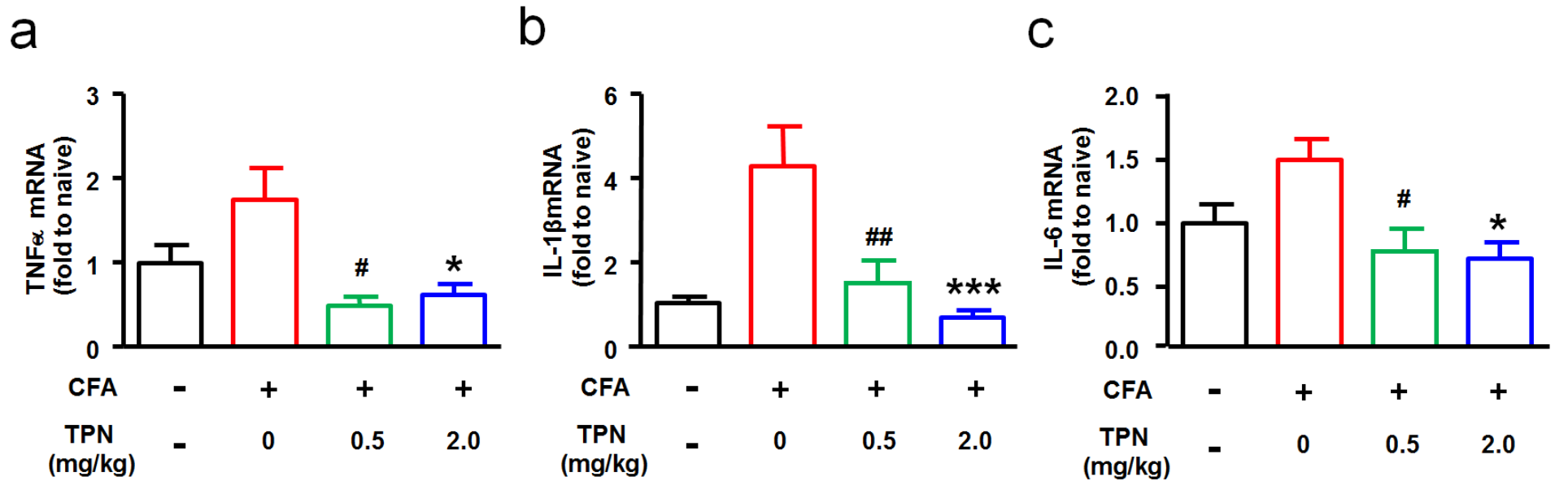

Figure 3

TPN decreases CFA-induced upregulation of TNF- $\alpha$, IL-1 $1 \beta$, and IL- 6 increases. (A-C) Effects of TPN on the mRNA expression levels of TNF- $\alpha(A), I L-1 \beta(B)$, and IL-6 (C). TPN treatment with both low dose $(0.5$ $\mathrm{mg} / \mathrm{kg})$ and high dose $(2 \mathrm{mg} / \mathrm{kg}$ ) decreased TNF-a, IL-1 $\beta$, and IL- 6 upregulation after CFA injection. $\mathrm{n}=5$ mice/group. \# $\mathrm{P}<0.05$, \#\# $\mathrm{P}<0.01$, low dose of TPN compared to CFA group; $\mathrm{P}^{\mathrm{P}}<0.05$, $* \star * P<0.001$, high dose of TPN compared to CFA group, one-way ANOVA. 

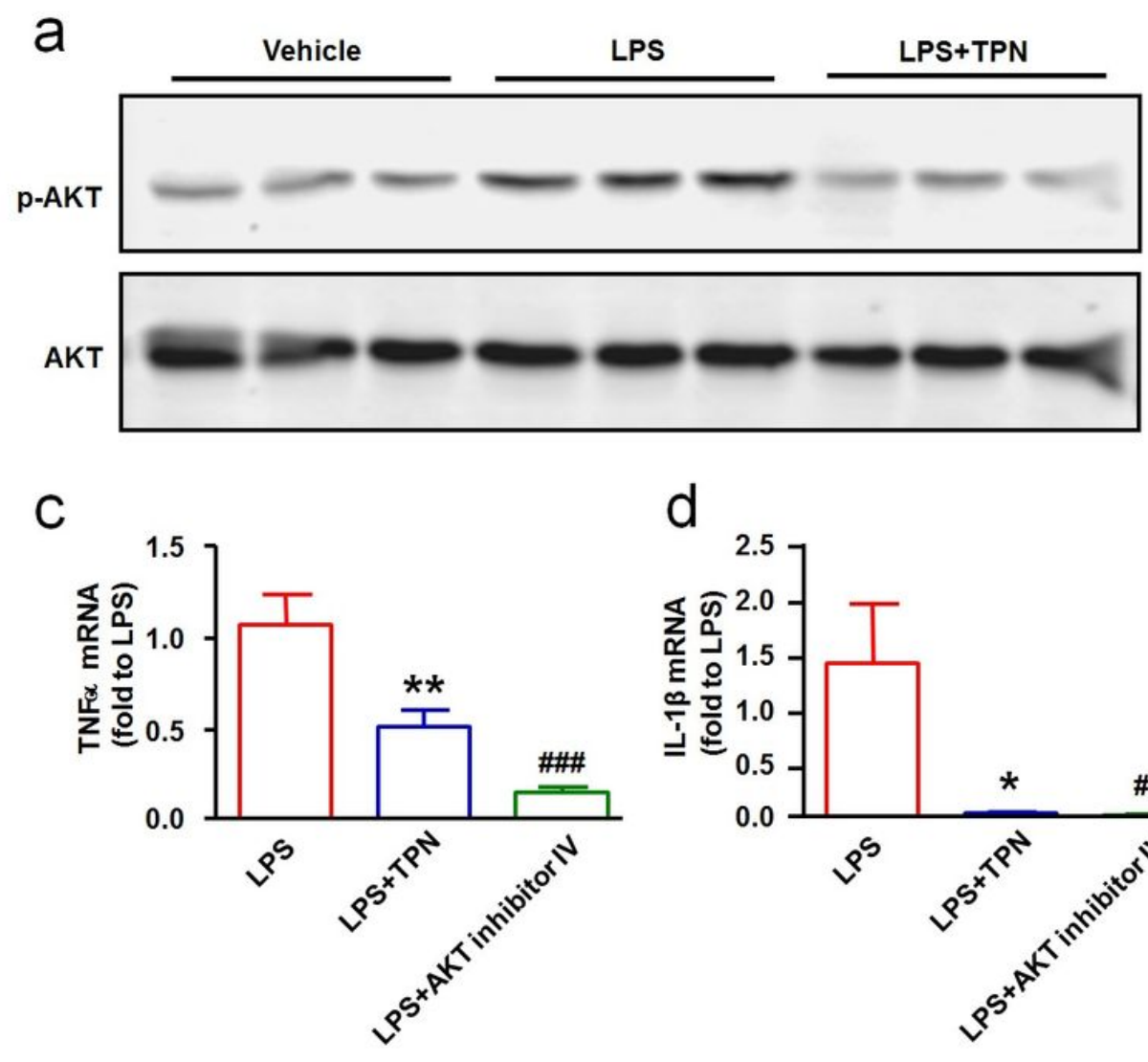

b
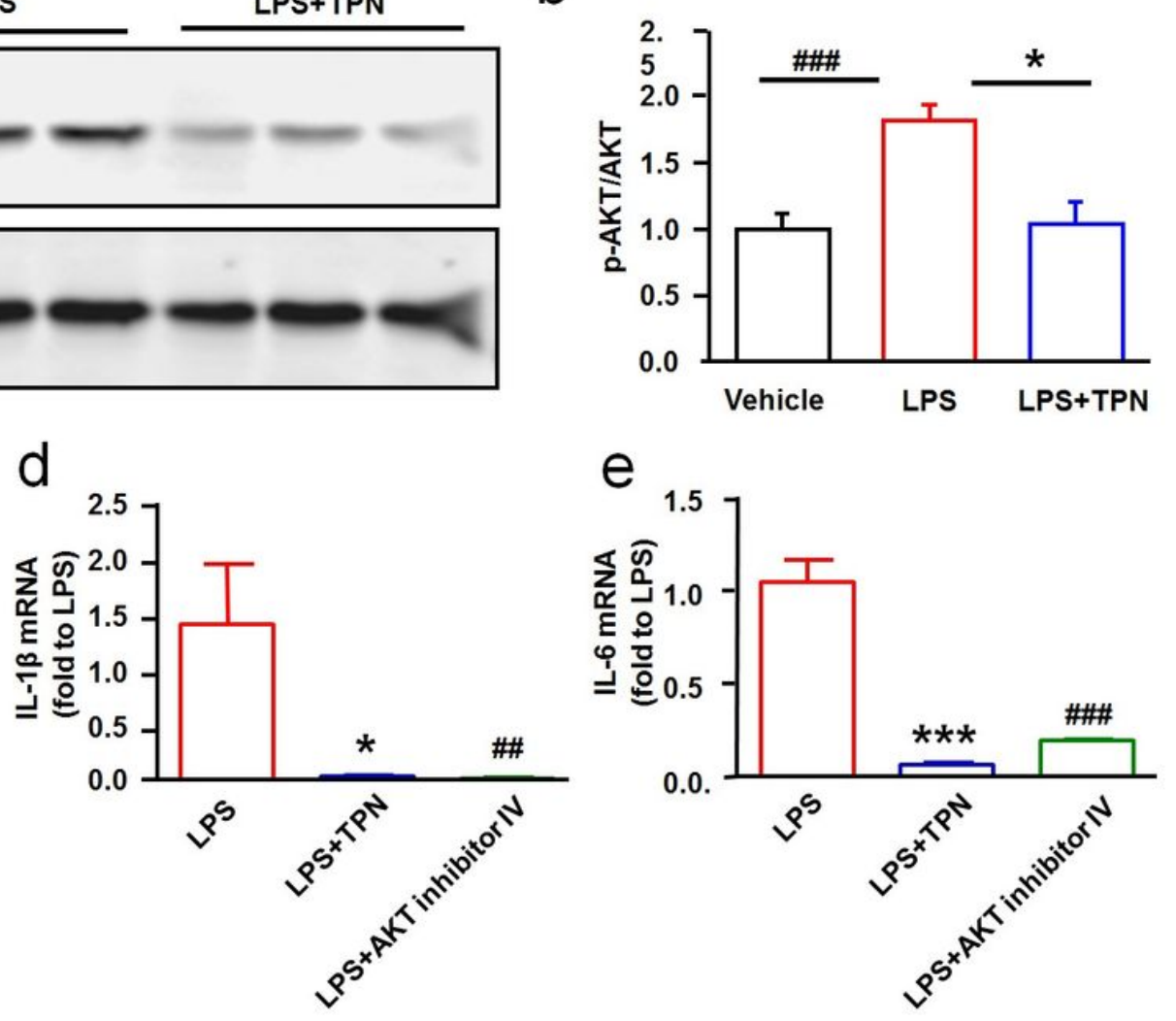

Figure 4

TPN decreases LPS-induced pAKT expression and the production of inflammatory cytokines in ND7/23 cells. (A and B) pAKT expression of LPS-induced ND7/23 Cells treated or untreated with TPN $(1 \mu \mathrm{g} / \mathrm{kg})$. pAKT expression was increased after LPS application and recovered in the LPS + TPN group. $n=3$. \#\#\# $P<0.001$, LPS + TPN group compared to Vehicle group; * $P<0.05$, LPS + TPN group compared to LPS group, one-way ANOVA; (C-E) Effects of TPN, and AKT inhibitor $₫$ on the mRNA expression levels of TNF-a (C), IL-1 $\beta$ (D), and IL-6 (E). Both TPN treatment $(1 \mu \mathrm{g} / \mathrm{mL})$ and AKT inhibitor $\otimes$ treatment $(1 \mu \mathrm{g} / \mathrm{mL})$ decreased TNF-a, IL-1 $\beta$, and IL- 6 upregulation after LPS incubation. $n=5 . * P<0.05$, ** $P<0.01$, *** $P<$ 0.001 , LPS group compared to the LPS + TPN group; \#\# $\mathrm{P}<0.01$, \#\#\# $\mathrm{P}<0.001$, LPS group compared to

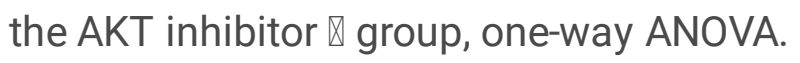


a

b
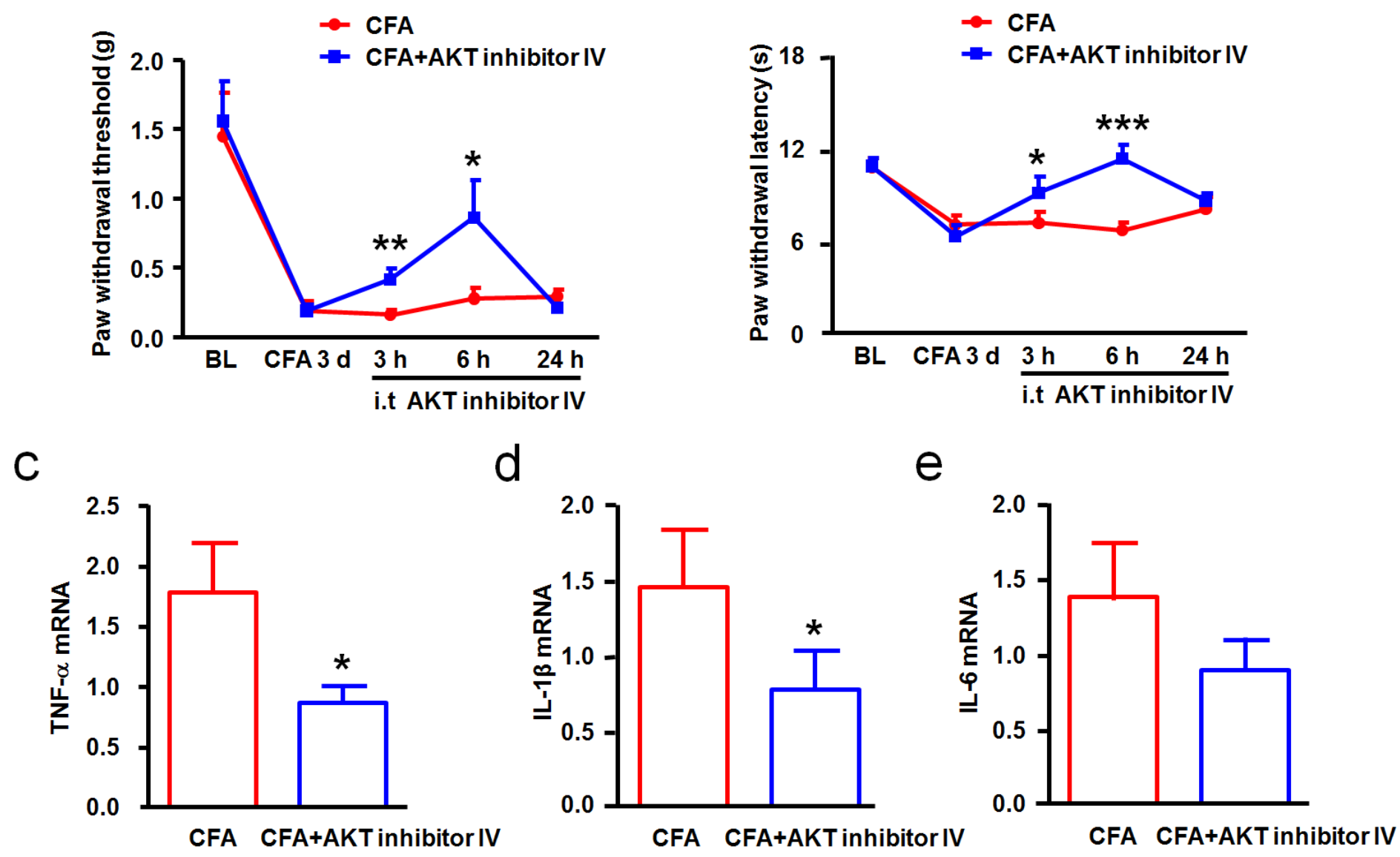

Figure 5

AKT inhibitor attenuates CFA-induced pain hypersensitivity and production of TNF- $a$, IL-1 $\beta$, and IL- 6 in the DRG. (A) AKT inhibitor $\otimes$ reversed CFA-induced mechanical allodynia at 3 and 6 hours after treatment. (B) The same treatment of AKT inhibitor $\otimes$ attenuated CFA-induced thermal hyperalgesia. ${ }^{*} \mathrm{P}<0.05$, $* * \mathrm{P}<$ $0.01, * \star * P<0.001$, compared with the CFA group. Two-way ANOVA followed by the Bonferroni test. (C-E) Intrathecal injection of AKT inhibitor $\otimes$ decreased expression of TNF-a, IL-1 3 , and IL-6 in the DRG. $n=6$. * $P<0.05, A K T$ inhibitor $\otimes$ group to the CFA group, Student's t-test.

\section{Supplementary Files}

This is a list of supplementary files associated with this preprint. Click to download.

- NC3RsARRIVEChecklistforNROSD1900272.pdf 